$========+$
N$^{\circ} 1$ VOL. I

Diciembre Revista INTEREDU

ISSN 2735-6523

2019

Investigación, Sociedad y Educación

https://doi.org/10.32735/S2735-65232019000180

57-73

\title{
CONVIVENCIA ESCOLAR Y FORMACIÓN CIUDADANA: PERCEPCIÓN DE LOS ESTUDIANTES DE SEGUNDO CICLO
}

School coexistence and citizen training from secondary students point of view

DANIEL ALBERTOSÁEZ SOTOMAYOR

Liceo Osorno College

dsaez@osornocollege.cl

LUIS ALFREDO AÑAZCO MARTíNEZ

Facultad de Deportes, Universidad Católica de Murcia

laanazco@alu.ucam.edu

\section{RESUMEN}

Este estudio busca evaluar la gestión de la calidad de la convivencia escolar en el marco de la inclusión, la democracia y las relaciones sanas. Este estudio consideró a 292 estudiantes de secundaria, de dos instituciones educativas de la ciudad de Osorno que presentaban un alto porcentaje de vulnerabilidad. Esta investigación tuvo un diseño de investigación cuantitativo, descriptivo y no experimental, que recopiló datos a través de la utilización de un cuestionario validado que se aplicó a estos estudiantes. Como principales resultados, el trato respetuoso de los maestros hacia los estudiantes es evidente. Hay apoyo para los estudiantes que presentaron necesidades especiales, así como un alto nivel de confianza entre los estudiantes y los maestros. Sin embargo, aunque las relaciones entre profesores y estudiantes estaban bien calificadas, quedó claro que también había ciertas diferencias en el trato. Además, no fue posible evidenciar la existencia de un trabajo colaborativo entre ellos que permita la mejora de la calidad de las relaciones sociales.

Palabras clave: Ciudadanía; convivencia escolar; democracia; formación ciudadana; inclusión.

\section{ABSTRACT}

This study seeks to evaluate the quality management of school coexistence in the framework of inclusion, democracy and healthy relationships. This study evaluated 292 secondary students, from two educational institutions from the city of Osorno which presented high percentage of vulnerability. This research required a quantitative, descriptive, non-experimental research design, which collected data through the application of a validated questionnaire which was applied to these students. As main results, respectful treatment from teachers to students is evident; there is support for students who presented special needs, and there is a high level of trust between students and 
teachers. However, although the relationships between teachers and students were well-qualified, it became clear that there were also certain differences in treatment. And in addition to that, it was not possible to evidence the existence of collaborative work between them which could allow the improvement of the quality of social relations.

Keywords: Citizenship; school coexistence; democracy; citizen training; students; inclusion.

\section{INTRODUCCIÓN}

Para lograr una interrelación positiva entre los miembros de una comunidad educativa, se debe promover el aprendizaje de la convivencia escolar y la formación ciudadana. Estas son acciones que requieren la participación de todos los integrantes, sin excluir a nadie. Los miembros de una comunidad escolar se desenvuelven en un espacio donde coexisten diversas personas que cumplen diversos roles y responsabilidades dentro del entorno educativo, conllevando al requerimiento de ciertos criterios y normas que permitan una coexistencia de respeto y armonía. Estas se deben promover por medio de la participación de cada uno de los integrantes de la comunidad. Es por ello que el trabajo realizado por el equipo de gestión en conjunto con el encargado de convivencia escolar debe propiciar un tipo de liderazgo abierto a la recepción de distintas opiniones y pensamientos que construyan el desarrollo del sentido de cohesión y sello en la comunidad educativa, permitiendo facilitar y promover el trabajo escolar (Sandoval, 2014).

Para el logro de esos objetivos es fundamental el diseño y ejecución de un plan de gestión de la convivencia escolar, en el que es necesario incorporar aspectos de la formación ciudadana. El clima y el aprendizaje de estos elementos deben estar orientados a representar una práctica de tipo habitual institucional en cada establecimiento. Esto permite ordenar, evaluar y modificar las acciones para el logro de objetivos en torno a las distintas necesidades de los establecimientos escolares, que logren la superación de las acciones aisladas o de nulo impacto (Ministerio de Educación de Chile [MINEDUC], 2015).

Este plan de gestión de la convivencia escolar requiere integrar las dimensiones de inclusión, democracia y educación para la paz que nos entregue las herramientas necesarias para la preparación de nuestros estudiantes en torno al desarrollo de una ciudadanía activa y participativa. El objetivo es una formación ciudadana que logre identificar estos factores como estructura de una coexistencia social y armónica de toda la comunidad (Organización de las Naciones Unidas para la Educación, la Ciencia y la Cultura [UNESCO], 2008).

58 | INTEREDU № 1 Vol. I (DiCIEMBRe 2019) PÁGS. 57-73. ISSN 2735-6523 


\section{CONVIVENCIA ESCOLAR Y FORMACION CIUDADANA}

La convivencia escolar y la formación ciudadana forman parte del proceso educativo, pues representan la vida compartida de la escuela. De tal modo, la convivencia para la ciudadanía es un requisito fundamental para la adquisición de un aprendizaje en sí mismo, que permite entender las características de las interrelaciones, manifestándose por medio de un ambiente propicio para crecer y aprender (Fierro et al., 2011; Fierro, 2013). Este tipo de cualificaciones son las que se asimilan a las dimensiones que analizamos: inclusiva, democrática y pacífica (UNESCO, 2008)

La convivencia inclusiva está orientada a generar un espacio de igualdad de oportunidades para todas las personas, siendo esta una condición que permite la inminente protección del derecho a la educación. Ella está direccionada a eliminar todo tipo de acción indebida hacia la diversidad, tales como: diversidad de género, origen cultural, religioso, condición de vida, necesidad educativa, entre otros (Fierro et al., 2011).

Respecto a la convivencia democrática, se refiere a la experiencia de las personas al participar en la vida compartida, vinculado al desarrollo de acciones reflexivas que permitan el trabajo colaborativo con otros. Además, teniendo la capacidad de resolución de problemas de una forma pacificadora, donde logren establecer acuerdos para la vida en común. La convivencia democrática debe permitir que el diálogo, comunicación, resolución de conflictos y el establecimiento de acuerdo sean formas necesarias de participación (Fierro, 2013).

En torno a la convivencia escolar como estructura fundamental para la ciudadanía y la democracia, es fundamental ampliar la atención en las regulaciones formales sobre la democracia, ya que el proceso de participación es observado como una oportunidad del desarrollo de la capacidad física (Fierro y Carbajal, 2019). La convivencia como educación permite abordar una oportunidad formativa escolar para aprender a reconocer y aportar en distintos sentidos como aportaciones cívicas. La convivencia pacífica enfoca a la educación como un derecho, que permite el respeto de los derechos sin discriminación o distinción por razones personales, de clase o grupo (Fierro et al., 2011).

Por lo tanto, es necesario prestar atención a la relevancia sobre la evaluación de la Convivencia Escolar y la Formación Ciudadana guarda. Por medio del documento de UNESCO se fundamenta la importancia de la Convivencia Escolar en las dimensiones democrática, inclusiva y de educación para la paz. En el presente se retoman la conceptualización realizada frente a la necesidad de tener un acercamiento a los procesos que intervienen en la convivencia y contar con un instrumento que permitan evaluar y describir el fenómeno de una manera objetiva (Fierro et al., 2011). 


\section{POLITICA NACIONAL DE CONVIVENCIA ESCOLAR}

La Convivencia Escolar se puede definir como el conjunto de las interacciones y relaciones que se desarrollan en una comunidad educativa entre todos los integrantes de la institución escolar (docentes, estudiantes, asistentes de la educación, directivos, padres, madres y apoderados y sostenedor). También forman parte los grupos, equipos, cursos y organizaciones que formen parte de la comunidad escolar, incluyendo también a las organizaciones que se encuentran insertas en el entorno (MINEDUC, 2019).

El MINEDUC presenta en su política nacional de convivencia escolar -en específico en el aspecto de inclusión- diversos cambios en materia educacional, cuyo objetivo es avanzar hacia una educación inclusiva y de calidad en todos los establecimientos escolares del país. Ello supone un cambio de paradigma, comprendiendo la educación como un derecho fundamental, considerando estándares de calidad integral destinados a promover la inclusión y la participación. También se indica que esta política tiene como finalidad la promoción y el resguardo de derechos con una mirada inclusiva, que permitan valorar y reconocer la diversidad y oportunidades de aprendizaje, brindando y garantizando la permanencia y calidad escolar de todos los estudiantes (MINEDUC, 2015).

En este contexto, la política nacional de convivencia escolar y de formación ciudadana se constituye como un marco orientador que recoge las aspiraciones más profundas de la reforma educacional. Su fin es potenciar el pleno desarrollo individual y social de los y las estudiantes, así como contribuir a la construcción de un país más justo, solidario y democrático. Sobre la gestión de la convivencia escolar, el MINEDUC menciona que debe ser coherente y pertinente, permitiendo saber el tipo de convivencia que se quiere promover de acuerdo al contexto de cada establecimiento y a sus actores educativos, con los objetivos y acciones que se diseñen e implementen (MINEDUC, 2019).

De este modo, el objetivo central de la política de convivencia escolar es orientar la definición e implementación de acciones, iniciativas, programas y proyectos que promuevan y fomenten la comprensión y el desarrollo de una convivencia escolar participativa, inclusiva y democrática, con un enfoque formativo, participativo, de derechos, equidad de género y de gestión institucional y territorial con un fuerte sentido de la educación para la paz (MINEDUC, 2015). La educación para la paz es necesaria concebirla como un proceso de desarrollo de la personalidad, que es continuo y permanente, inspirado en una forma positiva de aprender a vivir consigo mismo y con los demás en la no violencia y en la creación de ámbitos de justicia, de respeto y de armonía (González, 1993).

60 | INTEREDU № 1 VOL. I (DiCIEMBRE 2019) PÁGS. 57-73. ISSN 2735-6523 


\section{HACIA LA FORMULACIÓN DE LOS OBJETIVOS}

La convivencia escolar para la ciudadanía se presenta en la actualidad como un factor a considerar en el logro de resultados tanto institucionales como personales de los estudiantes. Es, en este sentido, que los establecimientos educativos están centrando sus esfuerzos en generar estrategias y formular proyectos que permitan que el clima escolar y la convivencia mejoren. Esto debería concluir en la formación de un ciudadano crítico, participativo y consciente de los procesos que ocurren tanto en el país como en el mundo.

Sabemos la importancia de una buena convivencia escolar y también sabemos que existen muchos aspectos para hablar de ella, por lo que cabe preguntarse: ¿Qué nivel de democracia existe en las escuelas? ¿Cuál es la importancia que se le da a la inclusión en los establecimientos? ¿Qué acciones se realizan para fomentar una cultura de paz dentro de los establecimientos? En consecuencia, la pregunta que orienta este estudio es: ¿Cómo se promueve desde la percepción estudiantil la convivencia escolar y la formación ciudadana, particularmente en sus dimensiones de inclusión, democracia y paz en los estudiantes de dos escuelas básicas municipales vulnerables de la comuna de Osorno?

Por tanto, como objetivo general del estudio se propone determinar la percepción de los estudiantes de dos escuelas básicas municipales vulnerables de la comuna de Osorno, acerca de la promoción de la convivencia escolar y la formación ciudadana en tres dimensiones propuestas por UNESCO: inclusión, democracia y paz.

Como objetivos específicos, se plantea primeramente determinar la percepción de los estudiantes de dos escuelas básicas municipales vulnerables de la ciudad de Osorno sobre de la convivencia escolar y la formación ciudadana respecto a la dimensión inclusión, declarada por la UNESCO. Como segundo objetivo específico, determinar la percepción de estos mismos estudiantes sobre de la convivencia escolar y la formación ciudadana respecto a la dimensión democracia declarada por la UNESCO. Como último objetivo específico, determinar la percepción de los estudiantes sobre de la convivencia escolar y la formación ciudadana sobre la dimensión educación para la paz, declarada por la UNESCO.

\section{DiSEÑO METODOLÓGICO DE LA INVESTIGACIÓN}

El enfoque de la investigación es de tipo cuantitativo, ya que se realiza la recolección de datos cuantitativos de una muestra, ya sea en forma aleatoria o discriminada, pero representativa de una población o fenómeno objeto de estudio (Tamayo, 2004). Es de carácter descriptivo porque busca especificar las características y los perfiles de personas, grupos comunidades o cualquier otro fenómeno que se someta a un análisis (Hernández, Fernández y Baptista, 2010). Por último, es un estudio de carácter no experimental, debido a que los fenómenos de estudio se observan en su contexto natural para luego ser analizados (Kerlinger y Lee, 2002).

INTEREDU № 1 VoL. I (DicIEMBRe 2019) PÁGS. 57-73. ISSN 2735-6523| 61 


\subsection{DETERMINACIÓN DE LA POBLACIÓN Y MUESTRA.}

La población fueron 299 estudiantes de dos establecimientos municipales de alta vulnerabilidad que cursaban segundo ciclo de Educación General Básica (EGB) en el año 2016. La muestra de estudiantes a quienes se les formularon las preguntas estuvo conformada por 292 estudiantes; esto es, el 97,4 \% del total de la población (ver Tabla 1). El $45 \%$ era estudiantes de género femenino y el 55\% masculino.

La técnica de muestreo utilizada en esta investigación es de tipo no probabilística (Parra, 2003). En relación a las preguntas del cuestionario, estas fueron aplicadas a los estudiantes de acuerdo a la concurrencia de ellos a sus clases presenciales. Para cada nivel adscrito al estudio se obtuvo una función discriminante estadísticamente significativa. En la Tabla 1 se presentan los principales resultados por nivel educativo.

Tabla 1. Población y muestra de estudiantes adscritos al estudio.

\begin{tabular}{|l|c|c|c|}
\hline \multicolumn{1}{|c|}{ CURSO } & POBLACIÓN & MUESTRA & $\begin{array}{l}\text { \% DE LA } \\
\text { MUESTRA }\end{array}$ \\
\hline Quinto Básico & 82 & 80 & 97,4 \\
\hline Sexto Básico & 75 & 72 & 95,7 \\
\hline Séptimo Básico & 70 & 68 & 96,9 \\
\hline Octavo Básico & 72 & 72 & 100 \\
\hline \multicolumn{1}{|c|}{ TOTAL } & 299 & 292 & 97,4 \\
\hline
\end{tabular}

Fuente: Datos obtenidos de libros de clase 2016.

\subsection{INSTRUMENTO UTILIZADO}

Para el presente estudio se utilizó una escala tipo Likert, validada por Chaparro et al. (2013) en la Universidad Autónoma de Baja California. Se realizó una adaptación del lenguaje y se aplicó a una muestra reducida ajustando dicho instrumento a las necesidades del estudio.

El instrumento final quedó integrado por 9 reactivos agrupados en las dimensiones de inclusión (4 reactivos), democracia (3 reactivos) y paz (2 reactivos). Para establecer su validez definitiva se sometió a juicio de experto obteniendo correcciones menores de formato. El instrumento tuvo una confiabilidad de 0,991.

\subsection{PROCEDIMIENTO}

Para la recogida de datos, primeramente se solicitó el consentimiento de participación por parte de las direcciones de las unidades educativas adscritas al estudio y de los estudiantes. Seguidamente, se comunicó a ellos el propósito de la investigación y lo 62 | INTEREDU № 1 VOL. I (DicIEMBRe 2019) PÁGs. 57-73. ISSN 2735-6523 
importante de sus respuestas para proponer mejoras educativas: lo fundamental para levantar propuestas con una base sólida de conocimientos e información. Una vez creado un ambiente cordial y de confianza se aplicó el instrumento.

El cuestionario fue aplicado por los investigadores y los docentes de cada curso, en las respectivas salas de clases de los alumnos y en las horas libres. Se ejecutó este procedimiento entre los meses de abril y agosto de 2016. El tiempo de duración de la aplicación del instrumento fue de 25 minutos aproximadamente. Una vez finalizada la recogida de los datos, se inició una labor exhaustiva de sistematización que confluye con la transcripción de los datos y su posterior análisis cuantitativo.

\section{PRESENTACIÓN Y ANÁLISIS DE LOS RESULTADOS}

\subsection{PERCEPCIÓN DE LOS ESTUDIANTES RESPECTO DE LA DIMENSIÓN INCLUSIÓN.}

\subsubsection{TRATO EQUITATIVO HACIA LOS ESTUDIANTES.}

Respecto del trato equitativo, el Gráfico 1 nos indica que los estudiantes percibieron que no existe un total acuerdo sobre el trato equitativo, ya que tan solo un $29 \%$ de los encuestados indica estar "totalmente de acuerdo", porcentaje que aumenta si sumamos a los estudiantes que indican en un $8 \%$ estar "bastante de acuerdo". Por otro lado, la mayor cantidad de estudiantes se sitúa en la opción "algo de acuerdo" en un 34\%, mientras que un $19 \%$ indica "algo en desacuerdo" y tan solo un 10\% indica "totalmente en desacuerdo".

Gráfico 1. Percepción de los estudiantes sobre el trato equitativo.

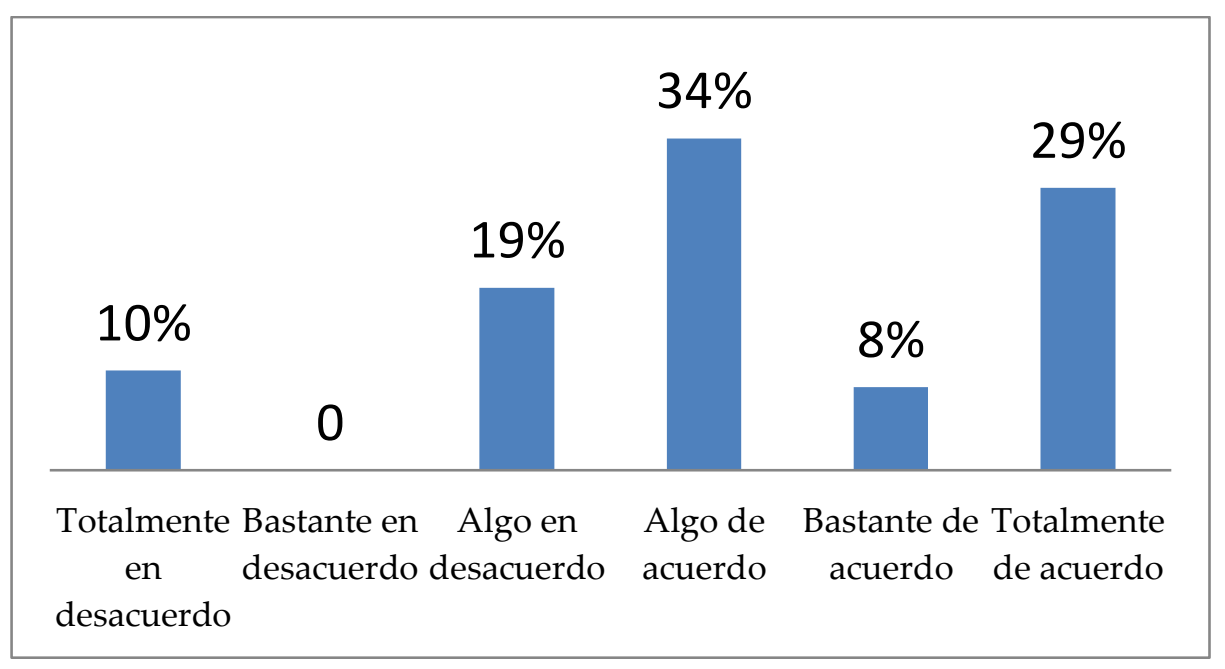

Fuente: Elaboración propia a partir de los datos obtenidos. 


\subsubsection{INTEGRACIÓN DE LAS NECESIDADES DISTINTAS DE LOS ESTUDIANTES.}

Respecto de la integración de las necesidades distintas de los estudiantes, el Gráfico 2 nos muestra que el 27\% indica estar "totalmente de acuerdo" y un 34\% manifiesta estar "bastante de acuerdo", cuestión que supera a los indicadores más bajos de la escala, ya que tan solo un 20\% indica estar en "algo en desacuerdo" con la afirmación, un 5\% "bastante en desacuerdo" y tan solo un $2 \%$ indica "totalmente en desacuerdo".

Gráfico 2. Percepción de los estudiantes sobre procesos inclusivos.

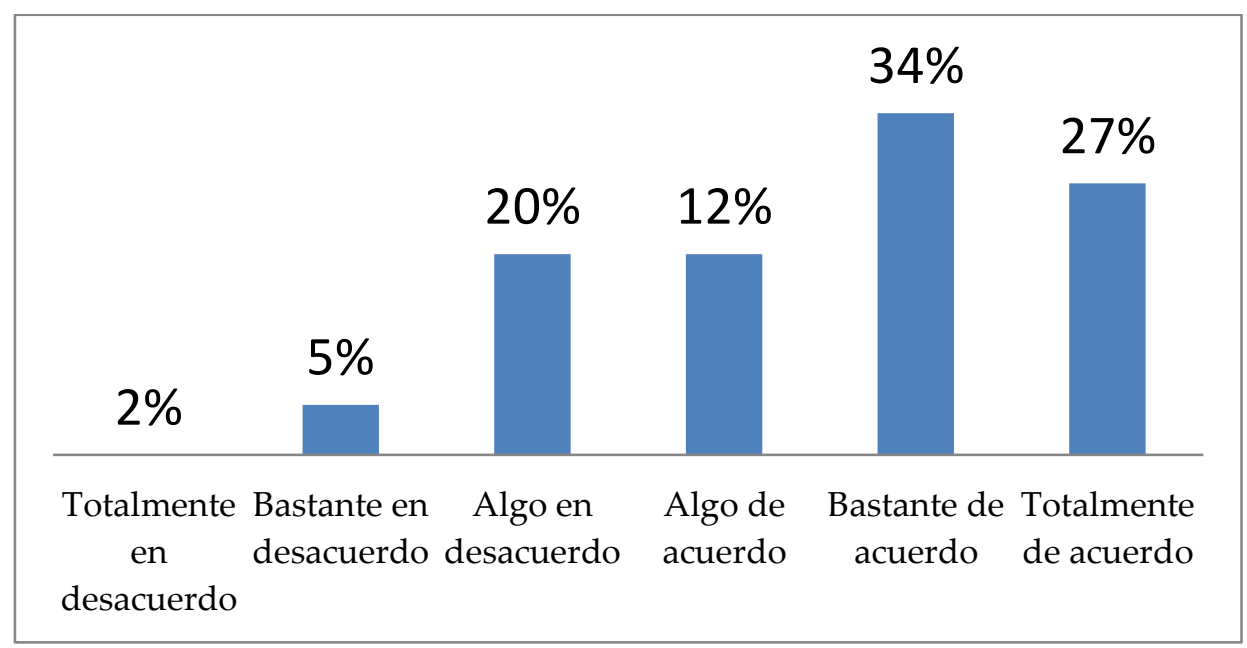

Fuente: Elaboración propia a partir de los datos obtenidos.

\subsubsection{ACCIONES DOCENTES DE BUEN TRATO y APOYO A ESTUDIANTES CON DiFICULTAD.}

En el Gráfico 3 se puede observar que esta afirmación en muy bien valorada por los estudiantes. Así es que un $40 \%$ de los encuestados indica estar "totalmente de acuerdo" con la afirmación. Esto, sumado al $24 \%$ que se inclina por estar "bastante de acuerdo". Por otro lado, un $21 \%$ de los estudiantes se encuentra "algo de acuerdo", mientras en la parte más baja de la escala se encuentra tan solo un $15 \%$ de las respuestas. 
Gráfico 3. Trato de los docentes a los estudiantes con dificultades.

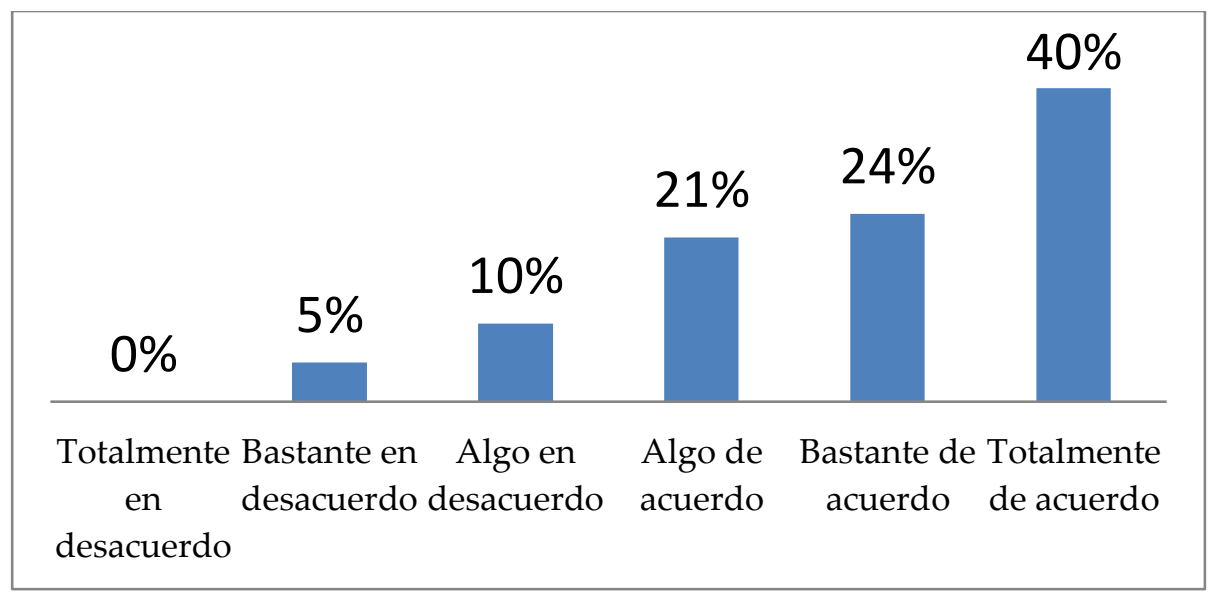

Fuente: Elaboración propia a partir de los datos obtenidos.

\subsubsection{IMPORTANCIA QUE SE LE ASIGNA AL TRABAJO COLABORATIVO.}

En el presente gráfico podemos observar que tan solo un $10 \%$ de los encuestados se manifiesta "totalmente de acuerdo" con esta afirmación, un 33\% se muestra "bastante de acuerdo", un 30\% se ubica en la medianía de la escala, indicando que está "algo de acuerdo". Por otra parte, un 10\% indica estar "algo en desacuerdo", un 7\% "bastante en desacuerdo" y un $10 \%$ indica estar "totalmente en desacuerdo".

Gráfico 4. Estrategia de trabajo utilizada por los docentes.

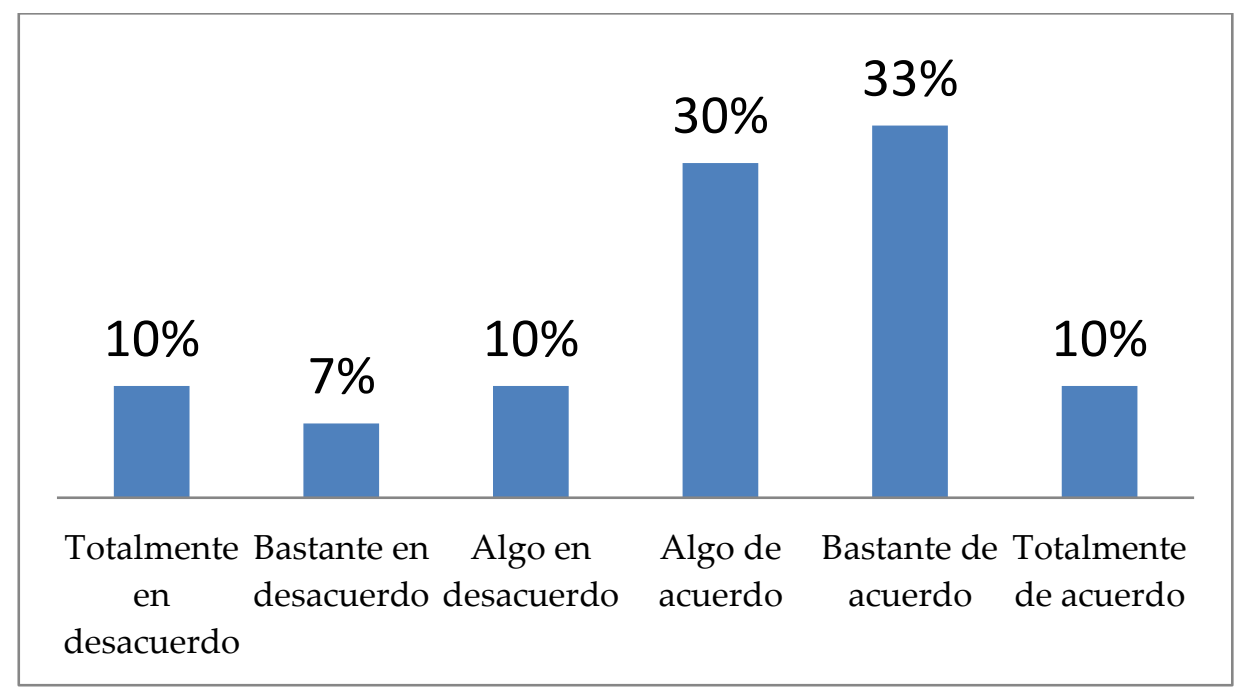

Fuente: Elaboración propia a partir de los datos obtenidos. 


\subsection{PERCEPCIÓN DE LOS ESTUDIANTES RESPECTO DE LA DIMENSIÓN DEMOCRACIA.}

\subsubsection{APLICACIÓN DEL REGLAMENTO DE FORMA EQUITATIVA.}

Todos los establecimientos educativos fijan sus normas de actuación y los plasman en sus manuales de convivencia escolar. Respecto de la aplicación de estas normas, los estudiantes encuestados indican tan solo en un 15\% estar "totalmente de acuerdo" con la aplicación justa de las normas, un 11\% indica estar "bastante de acuerdo" y un 48\%, "algo de acuerdo". Por otra parte, un 7\% de los encuestados indica estar "algo en desacuerdo", un 19\% "bastante en desacuerdo" y no se registraron respuestas en parte más baja de la escala.

Gráfico 5. Percepción de los estudiantes respecto de la aplicación de normas de manera equitativa.

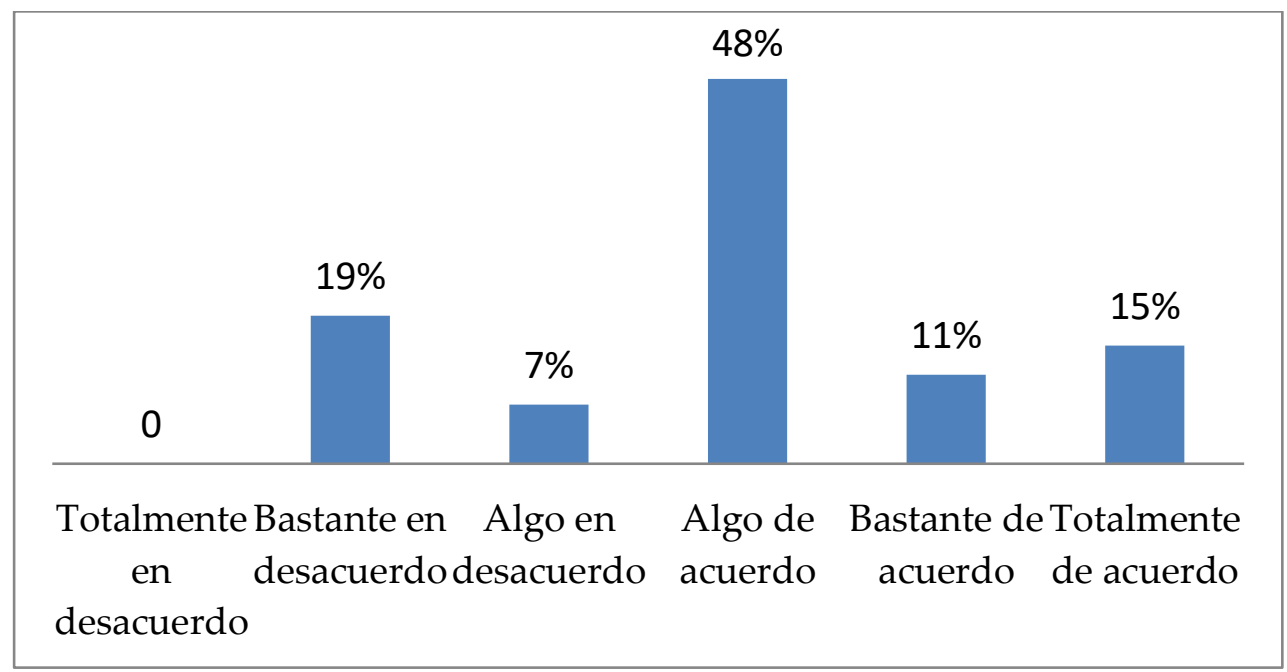

Fuente: Elaboración propia a partir de los datos obtenidos.

\subsubsection{PARTICIPACIÓN Y DIALOGO ENTRE LA COMUNIDAD.}

El presente grafico nos muestra que un $21 \%$ de los estudiantes encuestados indican estar "totalmente de acuerdo" con que existen oportunidades de participación y dialogo en el contexto escolar, misma cantidad de estudiantes que manifiestan "estar algo en desacuerdo". Lo que hace la diferencia es el 34\% de los estudiantes que indica estar "bastante de acuerdo" con la afirmación, mientras que un 14\% se muestra "algo de acuerdo" y un $10 \%$ manifiesta estar "totalmente en desacuerdo". 
Gráfico 6. Percepción de estudiantes sobre las oportunidades de diálogo que se generan.

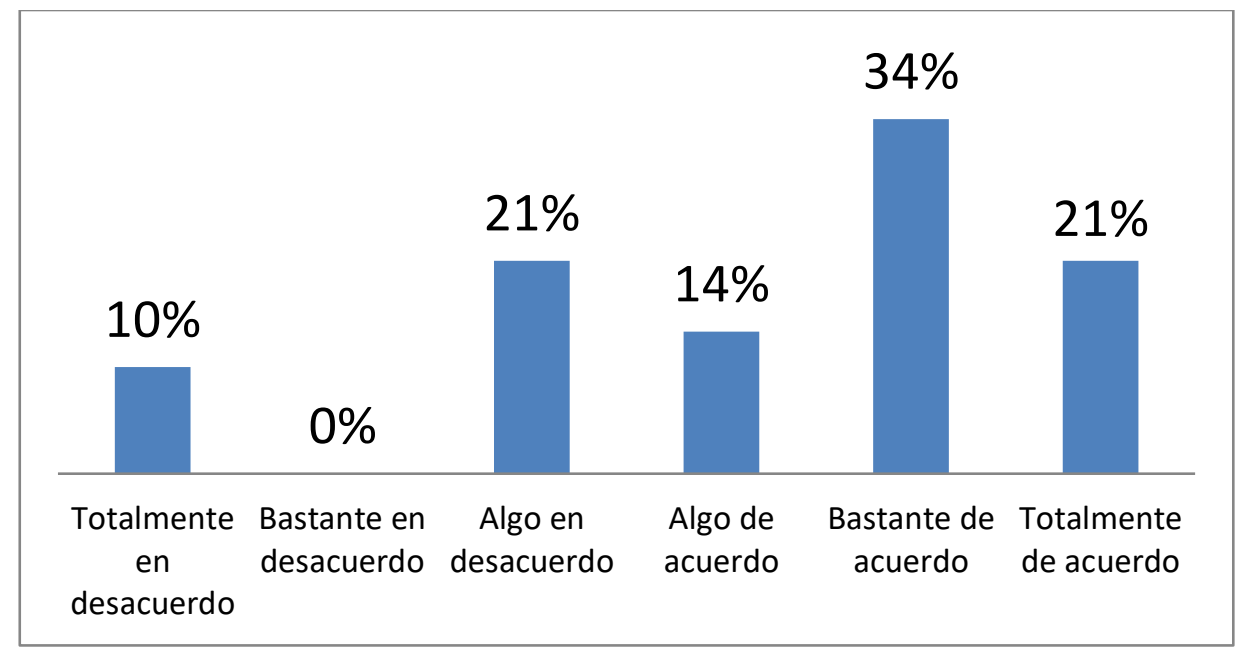

Fuente: Elaboración propia a partir de los datos obtenidos.

\subsubsection{RELACIÓN PROFESOR /ALUMNO.}

Cuando se les consulta a los encuestados respecto de la confianza mutua entre ellos y sus docentes, un 38\% indica estar "totalmente de acuerdo" con la existencia de esta, un $31 \%$ informa estar "bastante de acuerdo", y un 31\% "algo de acuerdo". Por otra parte, no existen estudiantes que ubiquen sus respuestas en las áreas más bajas.

Gráfico 7. Relación entre estudiantes y docentes en cuanto a la confianza entre las partes.

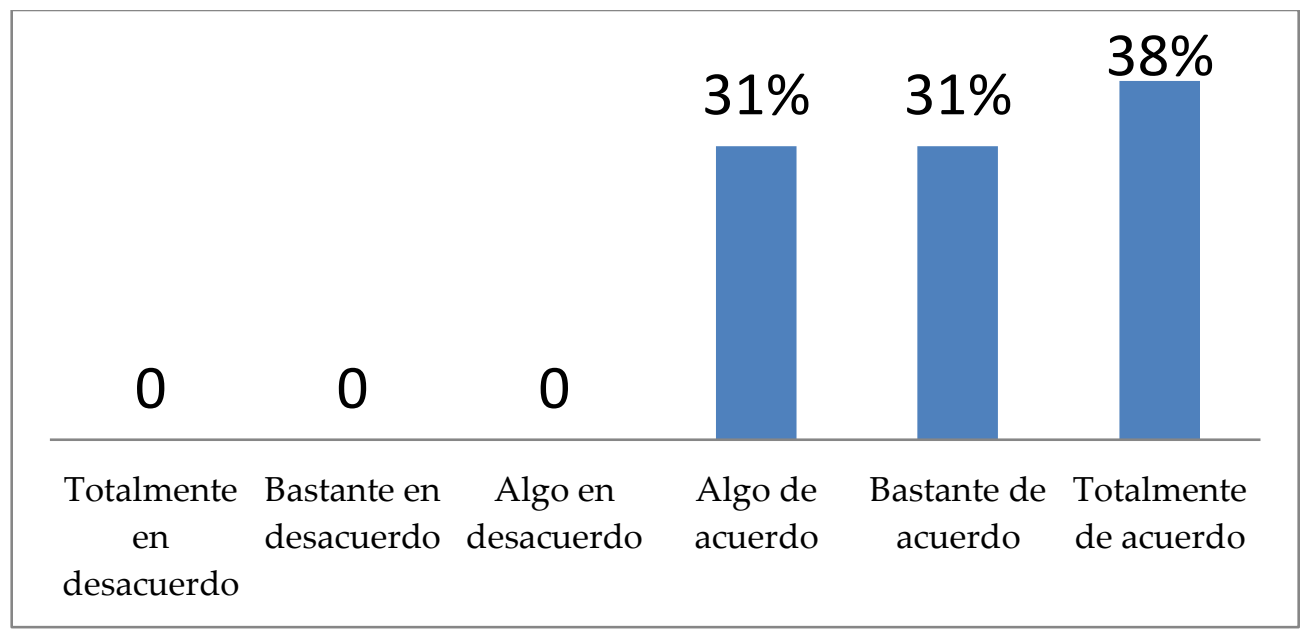

Fuente: Elaboración propia a partir de los datos obtenidos. 


\subsection{PERCEPCIÓN DE LOS ESTUDIANTES RESPECTO DE LA DIMENSIÓN PAZ.}

\subsubsection{ACTUAR DEL ESTABLECIMIENTO FRENTE A LAS CONDUCTAS DE RIESGO.}

El presente gráfico nos muestra que ante la afirmación "se observan acciones coordinadas para prevenir conductas de riesgo", un $22 \%$ de los estudiantes indica estar "totalmente de acuerdo", un 41\% manifiesta estar "bastante de acuerdo" y un 22\% indica estar "algo de acuerdo". Por otro lado, un 15\% de los estudiantes indica estar "algo en desacuerdo".

Gráfico 8. Acciones tomadas por el establecimiento ante conductas de riesgo.

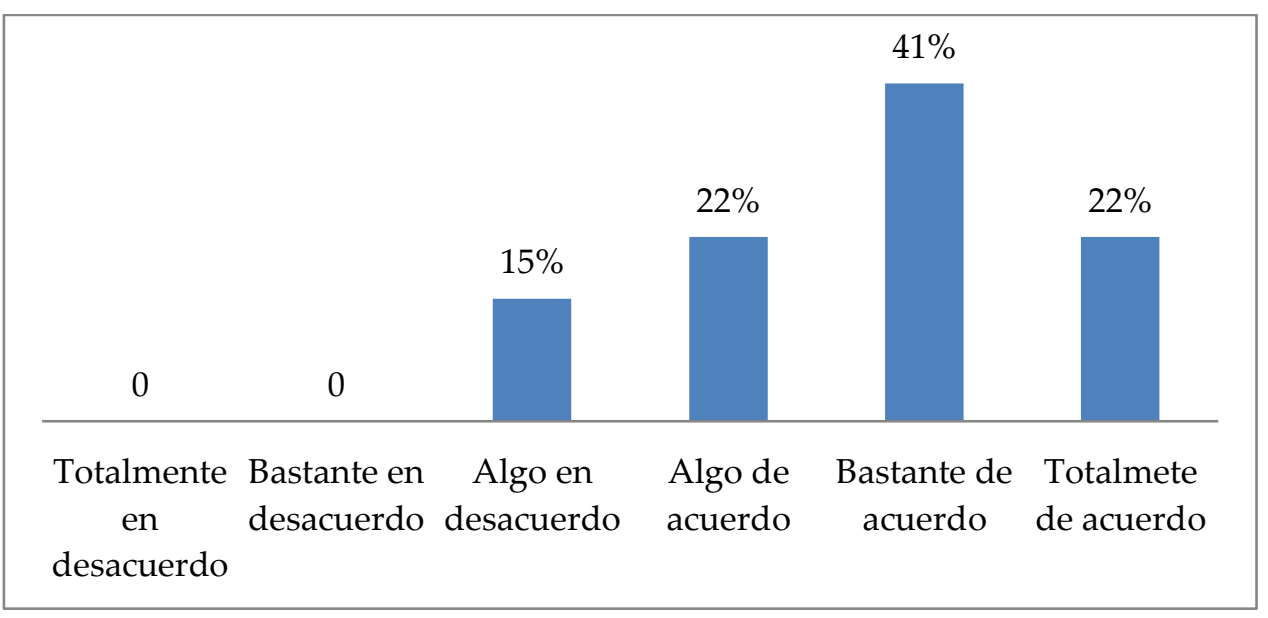

Fuente: Elaboración propia a partir de los datos obtenidos.

\subsubsection{DE LA COHESIÓN Y LA CONFIANZA ENTRE ESTUDIANTES.}

La confianza y la cohesión entre grupos es fundamental para el logro de objetivos educativos. Los estudiantes de los establecimientos investigados manifiestan en un $18 \%$ estar "totalmente de acuerdo" con que existe promoción de estos elementos, un 29\% se muestra "bastante de acuerdo" y un 18\% se manifiesta "algo de acuerdo". Como percepción negativa al reactivo, un 25\% manifiesta estar "algo en desacuerdo", un $7 \%$ indica estar "bastante en desacuerdo" y un 3\% muestra estar "totalmente en desacuerdo". 
Gráfico 9. Percepción de los estudiantes sobre la promoción de estrategias para unir a los estudiantes.

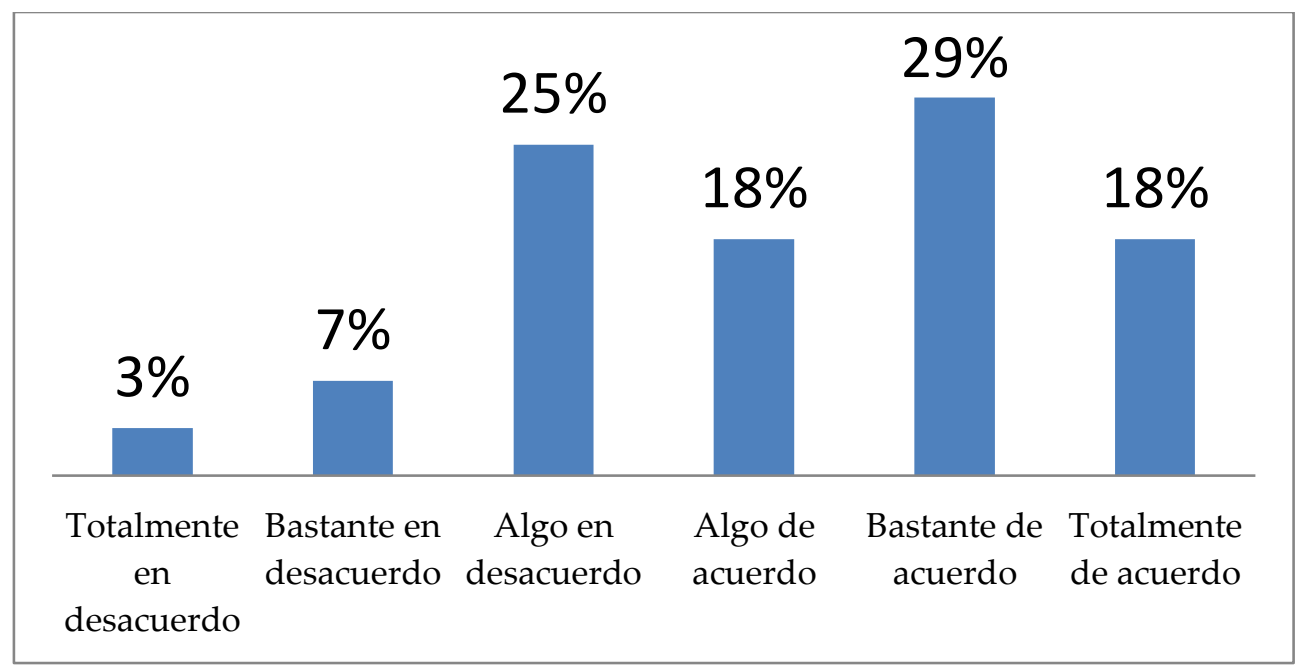

Fuente: elaboración propia a partir de los datos obtenidos.

\section{DISCUSIÓN}

La presente investigación se planteó como objetivo determinar la percepción de los estudiantes de dos escuelas básicas municipales vulnerables de la comuna de Osorno acerca de la promoción de la convivencia escolar y la formación ciudadana en tres dimensiones planteadas por la UNESCO: inclusión, democracia y paz.

Respecto a la dimensión Inclusión declarada por la UNESCO, los estudiantes perciben que esta dimensión se observa de manera positiva, aunque informan que en algunos aspectos esta inclusión puede ser mejorada, por ejemplo, en el ámbito de la equidad de trato. En relación con esto, un estudio sobre la percepción de los docentes en el trato con migrantes concluye que existen grandes avances respecto al trato positivo e inclusivo en la escuela (Pavez, 2019).

En Chile, la inclusión es un tema que se está comenzando a aplicar de forma efectiva hace un par de años por lo que para docentes como para estudiantes este es un tema nuevo que requiere un mayor estudio y análisis. El desafío de la inclusión en el país es posicionarse y declararse como un proyecto en la política cultural de la generación del conocimiento, como pasos necesarios para el logro de una educación y sociedad inclusiva (Manghi et al., 2020).

Por otro lado, y referente a la dimensión Democracia declarada por la UNESCO, la ley sobre violencia escolar indica que en materias de convivencia escolar y formación en ciudadanía, el reglamento interno del establecimiento educacional es el que debe incorporar las políticas y medidas a seguir de acuerdo a los protocolos establecidos en cada centro educativo. Respecto de la aplicación de estas normas, los 
resultados demostraron que un porcentaje de los estudiantes consideran que nos es justa la aplicación de las normas.

Este resultado coincide con un estudio que profundiza la problemática sobre las sensaciones de injusticia de los jóvenes en Buenos Aires, teniendo como resultados la injusticia en el trato diferente a estudiantes y la sanción como principal herramienta de control (Núñez, 2015). En relación a los resultados de percepción de estudiantes sobre las oportunidades de diálogo que se generan respecto de la confianza mutua entre ellos y sus docentes, los estudiantes manifiestan una percepción positiva. En torno a esto, en un estudio exploratorio por medio de una encuesta aplicada a 2711 estudiantes de primaria y secundaria de la ciudad de Querétaro, México, demostró que los factores asociados a las relaciones interpersonales maestro-alumno y los factores asociados a la enseñanza, son significativos para que se presenten situaciones que alteran la convivencia escolar (Cervantes y Vega, 2015).

Respecto de la dimensión Educación para la paz declarada por la UNESCO, se puede decir que las actividades que promueve el Fondo de las Naciones Unidas para la Infancia (UNICEF) siguen las directrices de los objetivos de desarrollo para el milenio y los principios de los derechos humanos. En este punto los estudiantes manifiestan en algunos aspectos una percepción positiva, pero en la mayoría de los casos, se muestra una tendencia hacia la indecisión y responden de forma afirmativa. En alusión a esto, un estudio a los docentes y estudiantes en el Colegio Maria Reina, de la ciudad de Medellín, describe las condiciones necesarias para crear y fortalecer iniciativas frente a la construcción de la paz, en donde por medio de las entrevistas, los estudiantes indican que las iniciativas de paz son necesarias y que su principal desarrollo es en la escuela (Quintero, 2018). Por lo tanto, es fundamental considerar el rol que cumple el profesor en torno a la promoción de la convivencia escolar y la formación ciudadana, en las dimensiones inclusión, democracia y paz en estudiantes.

\section{CONCLUSIONES}

Dando respuesta a los objetivos planteados en este estudio y a la luz de los resultados se puede concluir que respecto de la dimensión Inclusión declarada por la UNESCO, los estudiantes perciben una inclusión positiva, aunque también mencionan algunos aspectos a considerar, como es el caso del trato equitativo. En Chile, la inclusión es percibida por los docentes y estudiantes como un tema nuevo, que requiere un mayor estudio y análisis. De esta manera se podría generar en la ciudadanía una mayor preocupación y ocupación de estos acontecimientos.

70 | INTEREDU № 1 VOL. I (DiCIEMBRE 2019) PÁGS. 57-73. ISSN 2735-6523 
En torno a la dimensión Democracia declarada por la UNESCO, la ley sobre violencia escolar indica que en materias de convivencia escolar y ciudadanía, el reglamento interno deberá incorporar políticas de prevención, medidas pedagógicas, protocolos de actuación y una tipificación de las faltas y de las medidas, graduando las faltas de menor a mayor gravedad (leves, graves y gravísimas, como ejemplo). Además, agrega que en la aplicación de las medidas disciplinarias (que pueden ir desde una medida pedagógica hasta la cancelación de la matrícula) se deberá garantizar en todo momento el justo procedimiento. Pero esta ley no indica que los reglamentos y manuales permitan avanzar con esas medidas hacia la construcción de una mejor ciudadanía. Se concluye que lo que informan los estudiantes debe ser considerado a la hora de confeccionar estos planes.

Sobre este planteamiento podemos decir, que de las tres dimensiones evaluadas, la que se observa con mayor debilidad es la dimensión para la democracia, donde los estudiantes no concuerdan con que exista la aplicación justa de las normas, o no queda claro que el reglamento se aplique de manera justa. Esto repercutiría en el proceso de aprendizaje y adquisición de las actitudes, valores y creencias compartidas que intervienen en el proceso de configuración en los estudiantes de su condición de ciudadanos.

Respecto de la dimensión Educación para la paz declarada por la UNESCO, se puede decir que las actividades relacionadas con la educación para la paz que promueve la UNICEF siguen las directrices de los objetivos de desarrollo para el milenio y los principios de los derechos humanos. Estos principios implican un compromiso con la construcción de la paz, la reducción de las diferencias mediante medidas dirigidas a los niños más vulnerables, más marginados y más necesitados.

En este punto los estudiantes manifiestan en algunos aspectos una percepción positiva, pero en la mayoría de los casos se muestra una tendencia hacia la indecisión y responden de forma positiva. Los datos no muestran claridad para concluir que en los establecimientos adscritos al estudio se esté fomentando una educación para la paz, lo que a nuestro entender es el camino para la construcción de una ciudadanía consciente y para una educación centrada en el individuo y no tan solo centrada en la búsqueda de una calificación.

\section{REFERENCIAS BIBLIOGRÁFICAS}

Cervantes, O., y Vega, J. (2015). Percepción acerca de la interacción entre maestros y alumnos: su impacto en el bienestar de los estudiantes. Interacções, 11(38).

Chaparro, A., Caso Niebla, J., Díaz López C. (2013). Desarrollo y validación de un INTEREDU № 1 VoL. I (DiciembRe 2019) PÁGS. 57-73. ISSN 2735-6523| 71 
instrumento para la evaluación de la convivencia escolar. Actas del V Congreso Iberoamericano de violencia escolar. Santiago: Universidad Diego Portales.

Fierro, C. (2013). Convivencia inclusiva y democrática: Una perspectiva para gestionar la seguridad escolar. Sinéctica, (40), 01-18.

Fierro, C., Tapia, G., Carbajal, P., Bazdresch, M., Arias, E., Fortoul, B., Caso Niebla y Juárez, M. (2011). Instrumentos para el autodiagnóstico e intervención en escuelas basados en indicadores de convivencia democrática, inclusiva y no violenta. Protocolo de investigación no publicado.

Fierro, M.C. (2013). Convivencia democrática e inclusiva. Una perspectiva para gestionar la seguridad escolar. Revista Electrónica Sinéctica, 40, 1-18.

Fierro, C., y Carbajal, P. (2019). Convivencia Escolar: Una revisión del concepto. Psicoperspectivas, 18(1), 9-27.

González Lucini, F., (1993). Educación en valores y diseño curricular, Madrid, Alhambra Longmann.

Hernández, R., Fernández, C. y Baptista, P. (2010). Metodología de la Investigación.McGraw Hill.

Kerlinger, F. N. y Lee, H. B. (2002). Investigación del comportamiento. Métodos de investigación en ciencias sociales ( $4^{\mathrm{a}} \mathrm{ed}$.). México: McGraw-Hill.

Manghi, D., Solar, M., Ibarra, A., Godoy, I., Córdova,V., y Soto, K.. (2020). Para compreender a educação inclusiva chilena: panorama de políticas e pesquisa educativa. Cadernos de Pesquisa, 50(175), 114-135.

MINEDUC (2019). Política nacional de Convivencia Escolar. Santiago de Chile.

MINEDUC (2015). Política de convivencia escolar. Santiago de Chile.

Nuñez, P. (2015). Ideas de justicia en conflicto: Divergencias entre las regulaciones de los Acuerdos de Convivencia y las percepciones juveniles sobre la justicia en la escuela; Arizona State University; Education Policy Analysis Archives; 23; 57; 6-2015; 1-27.

Parra, J. (2003). Guía de muestreo. Editorial Faces, LUZ. Venezuela.

Pavez, I., Ortiz, J. y Domaica, A. (2019). Percepciones de la comunidad educativa sobre estudiantes migrantes en Chile: trato, diferencias e inclusión escolar. Estudios pedagógicos (Valdivia), 45(3), 163-183. https://dx.doi.org/10.4067/S071807052019000300163

Quintero, I. (2018). Percepciones de docentes y estudiantes sobre el desarrollo de iniciativas de paz en el Colegio María Reina del Carmelo de la ciudad de Medellín. Revista Interamericana de Investigación, Educación y Pedagogía, RIIEP, 11(2), 35-48.

Sandoval, M. (2014). Convivencia y clima escolar: claves de la gestión del conocimiento. Ultima década, 22(41), 153-178. https://dx.doi.org/10.4067/S071822362014000200007.

72 | INTEREDU № 1 VOL. I (DiCIEMBRE 2019) PÁGS. 57-73. ISSN 2735-6523 
Tamayo, M. (2004). El proceso de la investigación científica, (4ta ed). Editorial Limusa.

UNESCO (2008). Convivencia democrática, inclusión y cultura de paz: Lecciones desde la práctica educativa innovadora en América Latina. Recuperado de http://unesdoc.unesco.org/images/0016/001621/162184s.pdf 